\title{
Species Fractionation in Atomic Chains from Mechanically Stretched Alloys
}

\author{
Pedro Alves da Silva Autreto, ${ }^{1,2}$ Douglas S. Galvao, ${ }^{1}$ and Emilio Artacho ${ }^{2,3,4,5}$ \\ ${ }^{1}$ Institute of Physics Gleb Wataghin, University of Campinas, \\ UNICAMP Cidade Universitaria, 13083-970, Campinas, SP, Brazil \\ ${ }^{2}$ Department of Earth Sciences, University of Cambridge, Cambridge CB2 3EQ, United Kingdom \\ ${ }^{3}$ Theory of Condensed Matter, Cavendish Laboratory, \\ University of Cambridge, Cambridge CB3 OHE, United Kingdom \\ ${ }^{4}$ Nanogune and DIPC, Tolosa Hiribidea 76, 20018 San Sebastián, Spain \\ ${ }^{5}$ Basque Foundation for Science, Ikerbasque, 48011 Bilbao, Spain
}

(Dated: June 7, 2022)

\begin{abstract}
Bettini et al. [Nature Nanotech 1, 182 (2006)] reported the first experimental realization of linear atomic chains (LACs) composed of different atoms ( $\mathrm{Au}$ and $\mathrm{Ag}$ ). Different contents of $\mathrm{Au}$ and $\mathrm{Ag}$ were observed in the chains from what found in the bulk alloys, which rises the question of what is the wire composition if in equilibrium with a bulk alloy. In this work we address the thermodynamic driving force for species fractionation in LACs under tension, and we present density-functional theory results for $\mathrm{Ag}-\mathrm{Au}$ chain alloys. A pronounced stabilization of wires with an alternating $\mathrm{Ag}-\mathrm{Au}$ sequence is observed, which could be behind the experimentally observed $\mathrm{Au}$ enrichment in LACs from alloys of high Ag content.
\end{abstract}

\section{INTRODUCTION}

Metal nanowires have attracted great interest with the increasing miniaturization of electronic and mechanical devices $^{1}$, given their very interesting properties related to quantum transport ${ }^{2}$. From the experimental point to view, two techniques have been mostly used in nanowires research: mechanically controllable break junctions (MCBJ) $\sqrt{3 \sqrt[5]{5}}$ and in situ high-resolution transmission electron microscopy (HRTEM) $)^{511}$. While MCBJ is used to obtain electronic properties of nanowires, HRTEM gives structural information, providing atomic resolution with real-time image acquisition. A combination of both techniques is frequently used in order to establish a correlation between atomic arrangement and quantum conductance ${ }^{12}$. Since 1997 these techniques have revealed the existence of some quite unexpected structures, such as, extremely thin metal nanotubes with a square cross-section 13 , helical multishell gold nanowires 14 , single-walled metal nanotubes ${ }^{15 \mid 16}$, and the ultimate nanowires, linear atomic chains (LACs) $10[17$. The stability of these structures has been inferred using statistics over large number of observed structures (in HRTEM) and on conductance histograms for a large number of contact-breaking events (in MCJB). These experimental analyses have shown the remarkable existence of stable (or metastable) nanowires for alkali 33 and noble metals!

There have been many theoretical studies of these structures, mostly using classical atomistic models, as well as first principles ones $13 / 18,20$. Of particular importance is the work of Tosatti et al ${ }^{21}$, that investigated the stability of these nanostructures considering the metal wires connected to atomic reservoirs at both ends, determining the local minima (different metastable structures) as a function of the tension of the wire. Using this approach the authors were able to predict the stability of magic sizes for $\mathrm{Au}$ nanowires.
Although metal nanowires have been the object of intense theoretical and experimental investigations, most of these studies were carried out for pure metals. The first LAC experimental realization using metallic alloys was reported only in $2006^{22}$. They reported the observation of LACs of different lengths composed of $\mathrm{Ag}$ and $\mathrm{Au}$ atoms from $\mathrm{Ag}-\mathrm{Au}$ nanoalloys of different compositions. They observed that when the nanowires are rich in $\mathrm{Ag}^{22}$, in the final states of the stretching leading to LAC formation, the observed behavior was similar to the ones for $\mathrm{Au}$ rich nanowires 22 . From classical molecular dynamics 22 it was concluded that this unusual behavior is consequence of the $\mathrm{Au}$ enrichment at the region where the LACs were formed. The concentration of these nanoalloys changes significantly in time from the nominal initial values. They basically behave as if they had a much high Au content than in their nominal composition. This behavior was addressed by $\mathrm{Fad}^{23}$ by considering the enthalpy as defining the stability of these atomic chains under tension, in line with what pioneered by Tosatti21. A grand canonical perspective, however, which is the ad-

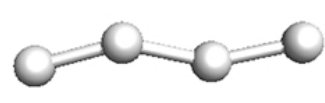

I

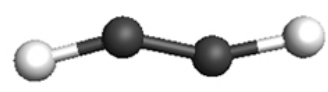

III

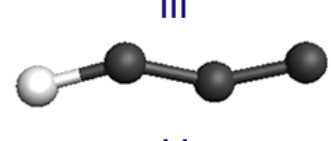

$\mathrm{V}$

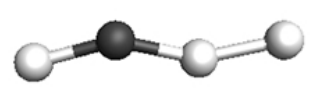

II

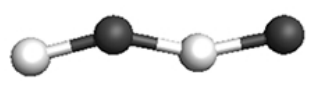

IV

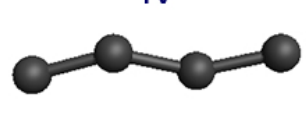

VI
FIG. 1. Structural motifs for the atomic-wire compositions considered in this work. Dark (light) balls indicate $\mathrm{Ag}(\mathrm{Au})$ atoms. See text for discussions. 
equate framework to study the thermodynamical trends in these systems, has not been done until this date and will be the focus of this work.

The main objective of this paper is to determine the relative concentrations of two species ( $\mathrm{Ag}$ and $\mathrm{Au}$ in this case) in LAC when in equilibrium with bulk alloys of given composition. For the purpose the relevant free energy is defined and calculated from first principles, analogously to what was done in ref. ${ }^{21}$ for single-species wires and tubes, which is now generalized to a grand-canonical setting. The observed capability of the wires to change composition with respect to the bulk reservoir demands such grand canonical treatment, beyond what was presented in ref.23.

In this way, with our approach we should be able to predict for specific bulk alloy concentrations what would be the corresponding likely concentrations for LACs formed by stretching a nanowire. It is important to remember, however, that this method takes an equilibrium viewpoint, which assumes atom exchange between reservoirs and wire, and should only be taken as a determination of equilibrium trends. The kinetics of the formation process will very probably originate deviations from this limiting behavior. It is however important to know what the thermodynamical trends are, which is what we address here.

\section{METHODOLOGY}

Density-functional theory (DFT) calculations were carried out for free-standing infinite nanowires by preparing arrays of sufficiently separated LACs in periodic boundary conditions. Tetragonal supercells are used with the wires aligned along the $x$-axis, with four atoms of $\mathrm{Au}$ or Ag and binary alloys of them. The considered geometries are presented in Fig. 11. The LAC geometries are relaxed for fixed supercell size, keeping the $y$ and $z$ sizes such that the minimum distance among wires is $15.0 \AA$, while the $x$ direction was varied in increments of $0.05 \AA$, from $6.00 \AA$ until the wire snapped. We have considered LAC lengths between $7 \AA$ and $11 \AA$.

The DFT calculations were performed with the SIESTA method 24125, using the Perdew-Burke-Ernzerhof (PBE) generalized gradient approximation (GGA) for exchange and correlation $\frac{26}{26}$ Norm-conserving scalar-relativistic pseudopotentials 27 replaced nuclei and core electrons, considering the normal core-valence separation for $\mathrm{Ag}$ and Au. Double- $\zeta$ polarized bases were used for valence electrons ${ }^{28}$. Integrals in real space were performed on a mesh of $250 \mathrm{Ry}$ cutofl ${ }^{25}$. The integration over the first Brillouin zone was carried out using the special $k$-points $\operatorname{method}^{29}$ with $k$-meshes of $1 \times 1 \times 30$ and $10 \times 10 \times 10$ for wire and bulk structures, respectively.

In order to calculate the nanowire stability for an alloy we have generalized the string tension analysis to different atomic species. The free energy $\Lambda$ we will seek to

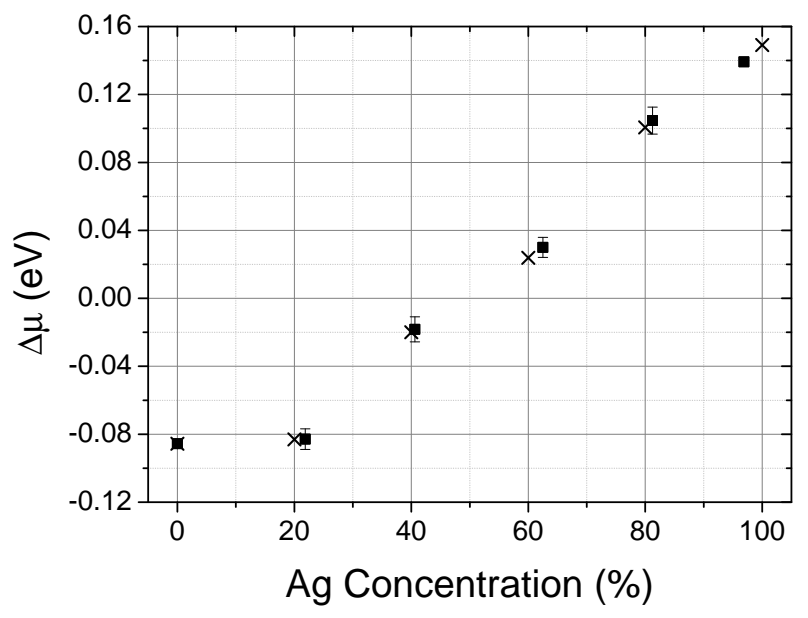

FIG. 2. Chemical potential difference $\Delta \mu=\left(\mu_{\mathrm{Au}}-\mu_{\mathrm{Ag}}\right) / 2$ calculated for a bulk $\mathrm{AgAu}$ alloy. Squares indicate $\Delta \mu$ values at bulk concentrations $0,7 / 32,13 / 32,20 / 32,26 / 32$ and 1 ; crosses show the interpolated estimates for concentrations 0 , $0.2,0.4,0.6,0.8$ and 1 .

minimize is

$$
\Lambda=E_{\mathrm{LAC}}-n_{\mathrm{Au}} \mu_{\mathrm{Au}}-n_{\mathrm{Ag}} \mu_{\mathrm{Ag}}
$$

where $n_{\mathrm{Au}}$ and $n_{\mathrm{Ag}}$ represent the number of $\mathrm{Au}$ and $\mathrm{Ag}$ atoms in the $\mathrm{LAC}$ (for the case of $\mathrm{AgAu}$ alloys), and $\mu_{\mathrm{Au}}$ and $\mu_{\mathrm{Ag}}$, are the corresponding chemical potentials. It is the zero-temperature grand-canonical free energy (per unit cell) that gives the stability for a wire of unit-cell length $L$ (length of cell along $x$ ). The analysis can also be done as a function of $F$, the force pulling the wire, in which case the relevant free energy would be ${ }^{21}$

$$
\Theta=\Lambda-F L .
$$

Here we will restrict ourselves to $L$-dependence and thus $\Lambda$. We will consider the low temperature limit, disregarding entropy effects. The generalization of the present scheme to finite temperature is costly but conceptually straightforward, either by including the entropy of harmonic vibrations ${ }^{30}$ (for a fully quantum albeit harmonic treatment), or thermodynamic integration for the free energy 31 , using molecular dynamics for classical nuclei, or with path-integral Monte-Carlo or molecular dynamics for quantum nuclei.

If we define

$$
n=n_{\mathrm{Au}}+n_{\mathrm{Ag}} ; \Delta n=n_{\mathrm{Au}}-n_{\mathrm{Ag}}
$$

we can write

$$
\Lambda=E_{\mathrm{LAC}}-n \bar{\mu}-\Delta n \Delta \mu
$$

where

$$
\bar{\mu}=\left(\mu_{\mathrm{Au}}+\mu_{\mathrm{Ag}}\right) / 2 \text { and } \Delta \mu=\left(\mu_{\mathrm{Au}}-\mu_{\mathrm{Ag}}\right) / 2 .
$$

The $n \bar{\mu}$ term controls the energetics related to their having more or less atoms in the chain, regardless of species, 
while the $\Delta n \Delta \mu$ term controls the energetics related to swapping atoms of different species in a wire with a given number of atoms. We will confine ourselves here mainly to the latter behavior considering wires with a constant $n$, and will consider the free energy

$$
\Omega=E_{\mathrm{LAC}}-\Delta n \Delta \mu=\Lambda+n \bar{\mu} .
$$

The chemical potentials are defined by the bulk alloy out of which the wire forms, which constitute the effective reservoirs. In particular, the relative chemical potential can be calculated as the statistical average energy change when swapping any $\mathrm{Au}$ atom in the alloy into a Ag atom,

$$
\Delta \mu=\langle\Delta E(\mathrm{Au} \rightarrow \mathrm{Ag})\rangle
$$

This has been obtained by making random substitutions of $\mathrm{Au}$ by $\mathrm{Ag}$ in disordered bulk alloy samples of different relative concentrations, relaxing the structures before and after the swap, and computing the energy difference. Face-centered cubic supercell structures with 32 atoms were used for the purpose. The original concentrations of $\mathrm{Ag}$ in $\mathrm{Au}$ were $0,7 / 32,13 / 32,20 / 32,26 / 32$, and 1 . The results are shown in Fig. 2. Both for $\mu_{\mathrm{Au}}$ and $\mu_{\mathrm{Ag}}$ the zero is defined for their respective pure bulk crystals. 32 The curve shows a quite linear behaviour except at very low Ag concentrations.

\section{RESULTS}

Fig. 3 shows the free energy $\Omega$ as a function of cell length $L$, for the six atomic-wire configurations shown in Fig. 1, and for the different bulk composition of the lead alloys specified on the figure. Starting from the left, the regime up to $\sim 7.7 \AA$ corresponds to zigzag wires in which three consecutive atoms define quite an equilateral triangle, i.e., essentially a band with two rows of atoms and edge-sharing triangles. The regime at larger distances corresponds to the zigzag structures shown in Fig. 1 , agreeing with what known for $\mathrm{Au}$ wires ${ }^{19}$, which gradually stretch with growing $L$, until they snap at $L$-values slightly above what displayed. The transitions between regimes, which involve "bond" breaking, are discontinuous and hysteretic, and thus will be quite dependent on quantum and thermal fluctuations .30 The experimentally observed LACs correspond to the intermediate regime.

Fig. 3 shows that for most alloy concentrations the structure IV of Fig. 1, with Ag-Au alternation is most stable in the LAC regime, with higher stabilization for higher Ag content in the bulk alloy. This implies that Agrich bulk alloys tend to produce wires with $[\mathrm{Ag}]$ tending towards $50 \%$, i.e. the wires will be Au enriched. These results agree with the mixed and Au enriched wires seen in the experimental atomically resolved micrographs. ${ }^{22}$ Reducing the Ag concentration in the alloy there is a transition to a situation in which the wire could change equilibrium concentration with stretching, as seen for $20 \%$. For $L \sim 9.5 \AA$, the wire would tend to pure $\mathrm{Au}$, thus

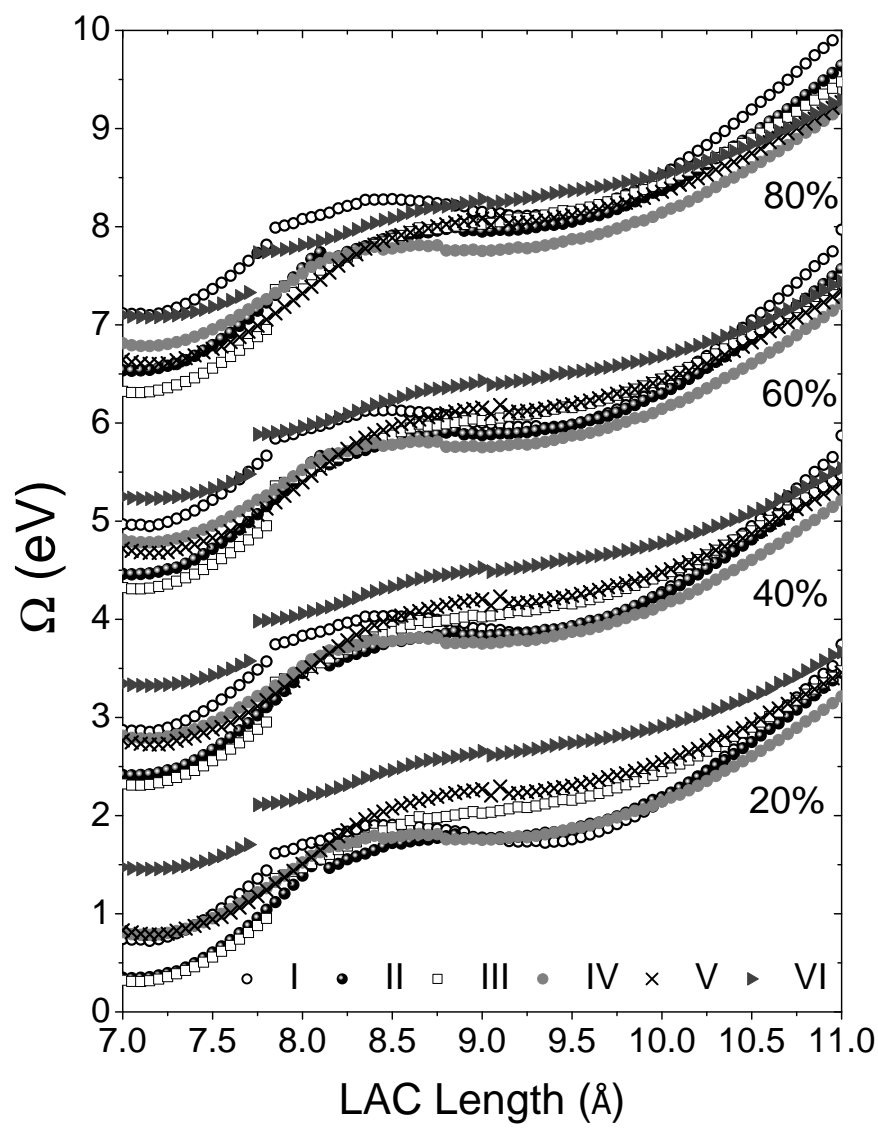

FIG. 3. Free energy $\Omega$ versus length $L$ for atomic chains of different compositions, for different bulk compositions. Each group of curves correspond to different bulk alloy composition, as specified with the percentages on the right, and contains six curves, for the wire configurations I to VI (see Fig. 1), as specified in the legend. The curves for different bulk compositions have been shifted upwards by $85.55 \mathrm{eV}$ for convenience.

depleting Ag (although the configuration II with $25 \% \mathrm{Ag}$ is close in energy, and indeed lower for mechanically unstable shorter $L$ ). Upon stretching, though, the AgAu alternating wire dominates again, thus replenishing Ag.

In summary, we have defined and calculated from firstprinciples the grand-canonical zero-temperature free energy governing the fractionation of two species between an alloyed atomic chain and the bulk alloy. We have applied this to $\mathrm{Ag} / \mathrm{Au}$ alloy chains and explained the tendency for $\mathrm{Au}$ enrichment in LACs observed experimentally for Ag rich alloys.

\section{ACKNOWLEDGMENTS}

PASA acknowledges the funding and support of the Erasmus Mundus program and the hospitality of the Department of Earth Sciences of the University of Cambridge. PASA and DSG acknowledge financial support of the Brazilian agencies FAPESP and CNPq. They also wish to thank Prof. Daniel Ugarte for many helpful dis- 
cussions. EA acknowledges the hospitality of the Institute of Physics at the University of Campinas. The cal- culations were done using the CamGrid high-throughput facility of the University of Cambridge.
1 J. Jia, D. Shi, J. Zhao, and B. Wang, Phys. Rev. B 76, 165420 (2007).

2 N. Agrait, A. L. Yeyati, and J. M. van Ruitenbeek, Phys. Rep. p. 81 (2003).

3 A. Yanson and I. Yanson, Nature 400, 144 (1999).

4 A. Mares and J. Van Ruitenbeek, Phys. Rev. B 72, 205402 (2005).

${ }^{5}$ V. Rodrigues, T. Fuhrer, and D. Ugarte, Phys. Rev. Lett. 85, 4124 (2000).

${ }^{6}$ Y. Kondo and K. Takayanagi, Phys. Rev. Lett. 79, 3455 (1997).

7 T. Kizuka, Phys. Rev. Lett. 81, 4448 (1998).

8 T. Kizuka, K. Yamada, S. Deguchi, M. Naruse, and N. Tanaka, Phys. Rev. B 55, 7398 (1997).

${ }^{9}$ H. Koizumi, Y. Oshima, Y. Kondo, and K. Takayanagi, Ultramicroscopy 88, 17 (2001).

10 H. Ohnishi, Y. Kondo, and K. Takayanagi, Nature 395, 780 (1998).

11 V. Rodrigues and D. Ugarte, Phys. Rev. B 6307 (2001).

12 M. Lagos, P. Autreto, D. Galvao, and D. Ugarte, J. Appl. Phys. 111, 124316 (2012).

13 M. Lagos, F. Sato, J. Bettini, V. Rodrigues, D. Galvao, and D. Ugarte, Nature Nanotechnology 4, 149 (2009).

14 Y. Kondo and K. Takayanagi, Science 289, 606 (2000).

15 Y. Oshima, H. Koizumi, K. Mouri, H. Hirayama, K. Takayanagi, and Y. Kondo, Phys. Rev. B 65, 121401 (2002)

16 Y. Oshima, A. Onga, and K. Takayanagi, Phys. Rev. Lett. 91, 205503 (2003).

17 P. Coura, S. Legoas, A. Moreira, F. Sato, V. Rodrigues, S. Dantas, D. Ugarte, and D. Galvão, Nano Letters 4, 1187 (2004).
18 A. Nakamura, M. Brandbyge, L. Hansen, and K. Jacobsen, Phys. Rev. Lett. 82, 1538 (1999).

19 D. Sánchez-Portal, E. Artacho, J. Junquera, P. Ordejón, A. García, and J. Soler, Phys. Rev. Lett. 83, 3884 (1999).

20 R. Senger, S. Dag, and S. Ciraci, Phys. Rev. Lett. 93, 196807 (2004).

21 E. Tosatti, S. Prestipino, S. Kostlmeier, A. Dal Corso, and F. Di Tolla, Science 291, 288 (2001).

22 J. Bettini, F. Sato, P. Coura, S. Dantas, D. Galvão, and D. Ugarte, Nature Nanotechnology 1, 182 (2006).

23 W. Fa and J. Dong, J. Chem. Phys. 128, 244703 (2008).

24 P. Ordejón, E. Artacho, and J. Soler, Phys. Rev. B 53, 10441 (1996).

25 J. Soler, E. Artacho, J. Gale, A. García, J. Junquera, P. Ordejón, and D. Sánchez-Portal, Journal of Physics: Condensed Matter 14, 2745 (2002).

26 J. Perdew, K. Burke, and M. Ernzerhof, Phys. Rev. Lett. 77, 3865 (1996).

27 N. Troullier and J. Martins, Phys. Rev. B 43, 1993 (1991).

28 E. Anglada, J. M. Soler, J. Junquera, and E. Artacho, Phys. Rev. B 66, 205101 (2002).

29 H. Monkhorst and J. Pack, Phys. Rev. B 13, 5188 (1976).

30 E. Hobi Jr, A. Fazzio, and A. da Silva, Phys. Rev. Lett. 100, 56104 (2008).

31 D. Frenkel and B. Smit, Understanding molecular simulation: from algorithms to applications, vol. 1 (Academic Pr, 2002).

32 This is for convenience with the numbers. The bare $\Delta \mu$ coming from $\Delta E^{\mathrm{DFT}}(\mathrm{Au} \rightarrow \mathrm{Ag})$ gives large numbers referring to the separate cores and electrons. Here we use $\Delta E^{\mathrm{DFT}}(\mathrm{Au} \rightarrow \mathrm{Ag})+\mu_{\mathrm{Au}}^{b}-\mu_{\mathrm{Ag}}^{b}$, the two latter terms being the DFT energy per atom of bulk Au and bulk Ag, respectively. The same is done in the definition of $E_{\mathrm{LAC}}$. 